\title{
SENSORIAMENTO REMOTO COMO SUBSÍDIO À MODELAGEM DO BALANÇO ENERGÉTICO SUPERFICIAL
}

\author{
Vassiliki Terezinha Galvão Boulomytis ${ }^{1}$ \\ ${ }^{1}$ Divisão de Sensoriamento Remoto/Instituto Nacional de Pesquisas Espaciais - INPE \\ likitgb@dsr.inpe.br
}

\section{RESUMO}

O balanço energético superficial interfere no funcionamento do sistema terrestre através da relação existente entre a água e a energia solar e do modo como ocorre o seu processo de partição. Para a sua estimativa, modelagens vêm sendo realizadas utilizando-se de um ou mais sensores com diferentes resoluções, a fim de se obter dados com resoluções temporal e espacial adequadas a uma escala local. Neste estudo serão apresentados modelos para estimar a evapotranspiração e outros parâmetros biofísicos através do balanço energético superficial com a utilização dos sensores MODIS e AMSR-E.

Palavras-Chave: Fluxo de calor, saldo de radiação, evapotranspiração, sensor MODIS, sensor AMSR-E.

\section{REMOTE SENSORS AS SUBSIDY TO THE MODELLING OF THE SUPERFICIAL ENERGY}

\begin{abstract}
The superficial energy balance interferes in the functioning of the land surface system because of the relationship between the water and the solar energy and as the partition process occurs. In order to estimate it, modelings have been done by the use of one or more sensors with different resolutions, in order to obtain data with satisfactory time and spatial resolutions for a local scale. In this study evapotranspiration and other biophysical parameter models will be presented regarding the superficial energy balance by the use of MODIS and AMSR-E sensors. Key Words: Heat flux, net radiation, evapotranspiration, MODIS sensor, AMSR-E sensor.

\section{INTRODUÇÃO}

A água e a energia solar são essenciais para a manutenção da vida terrestre. Os seus processos de partição, ou seja, o ciclo hidrológico e o ganho energético encontram-se fortemente relacionados e não podem ser tratados de modo isolado. $\mathrm{O}$ balanço de energia superficial determina a quantidade de fluxo energético disponível para evaporar a água e aumentar ou diminuir a temperatura superficial (Hartmann, 1994). Segundo Chapin III et al. (2002), todos os ciclos biogeoquímicos são controlados pelos ciclos hidrológicos, influenciando os seus processos bióticos, dissolvendo nutrientes e transferindo os mesmos entre os diversos ecossistemas. Assim sendo, todas as intervenções no ciclo hidrológico podem afetar o funcionamento dos ecossistemas terrestres e por isso tornaram-se um amplo campo de estudos para pesquisadores de diversas áreas.
\end{abstract}


As estimativas indiretas de evapotranspiração, através da medição dos fluxos de energia, calor sensível e latente, ou as medições diretas utilizando-se de lisímetros, de massa ou de drenagem, balanço de umidade do solo, ou evaporímetros, demandam um longo prazo para a sua execução e proporcionam respostas pouco efetivas com comportamento pontual, inviabilizando a aquisição sistemática de dados (Ferraz et al., 2009).

Giarolla \& Sano (2006) relatam que radiômetros passivos e orbitais com operação na faixa espectral de microondas vêm sendo utilizados para a estimativa de parâmetros biofísicos e oferecem resultados com erro quadrático médio baixo e aceitável, da ordem de 1 a $2 \%$. Dentre os sensores mais utilizados para a estimativa de parâmetros biofísicos estão o Advanced Microwave Scanning Radiometer (AMSR-E) e o Moderate Resolution Imaging Spectroradiometer (MODIS), da plataforma Aqua do Earth Observation System (EOS). O escopo principal deste estudo é de realizar uma revisão bibliográfica, abordando os modelos recentes utilizados para a estimativa do balanço energético superficial e de parâmetros associados ao mesmo com o auxílio das técnicas e dos instrumentos atualmente empregados no sensoriamento remoto.

\section{DESENVOLVIMENTO}

\section{Modelos de balanço energético superficial}

Em um dia claro, a radiação direta do sol constitui $90 \%$ da entrada de ondas curtas ao ecossistema. A parte adicional de ondas curtas vem como radiação difusa, que é espalhada por partículas e gases na atmosfera, ou então como radiação refletida das nuvens e da paisagem de entorno, tais como lagos e campos. Em um dia nublado ou ao entardecer, a radiação difusa se torna proporcionalmente maior, ao contrário da radiação direta. Portanto, no sistema terrestre como um todo, as radiações direta e difusa contam com aproximadamente metade da radiação de ondas curtas de entrada. A proporção da radiação de ondas curtas de entrada absorvida depende do albedo $(\alpha)$ ou da reflectância superficial de ondas curtas (Chapin III et al., 2002).

De acordo com Ferraz et al. (2009), o balanço energético superficial é proporcional ao saldo de radiação $\left(R_{n}\right)$. Rosenberg et al. (1983) afirmam que o saldo de radiação é a diferença entre os fluxos de radiação de ondas curtas e longas, que incidem e são refletidas da superfície, conforme a Equação 1:

$R_{n}=R_{s w b a l}+R_{l w b a l}=(1-a)\left(R_{s w \downarrow}\right)+\left(R_{l w \downarrow}-R_{l w \uparrow}\right)\left[W m^{-2}\right](1)$

onde: $R_{\text {swbal }}$ é o balanço de radiação de ondas curtas; $R_{l w b a l}$ é o balanço de radiação de ondas longas; a é o albedo ou refletividade; $R_{s w \downarrow}$ ) é o fluxo de radiação de ondas curtas incidente; $R_{l w \downarrow}$ é o fluxo de radiação de ondas longas incidente; $R_{l w \uparrow}$ é o fluxo de radiação de ondas longas refletido.

Rosenberg et al. (1983) e Chapin III et al. (2002) relatam que o saldo de radiação indica a quantidade fundamental de energia disponível na superfície da terra para conduzir os processos de evapotranspiração, aquecimento do solo e do ar. Além disso, também conduz a fotossíntese, que é o processo de menor consumo energético, mas essencial para a manutenção do ciclo de carbono. O saldo de energia é constituído pelos três maiores agentes de troca de energia entre o ecossistema e a atmosfera, ou seja, o fluxo do calor no solo $(G)$, o fluxo de calor latente ou evapotranspiração $(\lambda E)$, e o fluxo do calor sensível $(H)$, como é possível observar na seguinte Equação 2:

$$
R_{n}=G+\lambda E+H\left[W^{-2}\right](2)
$$

Friedl (2002) afirma que para a maioria dos estudos de balanço energético, baseados em técnicas de sensoriamento remoto, o $R_{n}$ 
é conhecido ou pode ser facilmente calculado. Quanto ao G, o mesmo pode ser calculado em função da proporção com $R_{n}$, segundo a Equação 3 desenvolvida por Bastiaanssen (1995):

$G / R_{n}=T / \alpha\left(0,0038 a+0,0074 \alpha^{2}\right)\left(1-0,98 N V D I^{4}\right)$

onde: $T_{s}$ é a temperatura da superfície radiométrica; $\alpha$ é o albedo; $N V D I$ é o índice de vegetação da diferença normalizada.

Os termos $\lambda E$ e $H$ referem-se às quantidades de fluxo turbulento e dependem de uma série de outras variáveis para serem estimados. Por convenção, os mesmos são modelados com base na analogia à Lei de Ohm, como consta nas Equações 4 e 5:

$$
\begin{gathered}
H=\frac{\rho C_{p}\left(T_{r}-T_{a}\right)}{r_{a}} \quad\left[W^{-2}\right] \\
\lambda E=\frac{\rho C_{p}\left(e_{o}-e_{a}\right)}{\gamma\left(r_{v}+r_{a}\right)}\left[W^{-2}\right]
\end{gathered}
$$

onde: $\rho$ é a densidade do ar; $C_{p}$ é o calor específico do ar; $T_{r}$ é a temperatura de referência; $T_{a}$ é a temperatura na camada atmosférica; $r_{a}$ é a resistência aerodinâmica; $e_{o}$ é a pressão de vapor; $e_{a}$ é a pressão de vapor na camada atmosférica; $r_{v}$ é a resistência estomática; $\gamma$ é a constante psicrométrica. Para estimar os componentes do fluxo turbulento, a temperatura da superfície radiométrica obtida através do sensoriamento remoto $\left(T_{s}\right)$ substitui a $T_{r}$ da Equação 4 (Renzullo et al., 2008). O parâmetro $\lambda E$ é freqüentemente estimado como o resíduo da equação de balanço energético baseado nas estimativas de $H$, $G$, e $R_{n}$ (Friedl, 2002).

\section{Dados relevantes dos Sensores MODIS e ASMR-E}

O objetivo do sensor MODIS é de quantificar e detectar as mudanças da cober- tura terrestre auxiliando os diversos modelos existentes. Os dados são transferidos para as estações terrestres e então enviados para processamento nos sistemas de operações. Estes são divididos em cinco níveis (de 0 a 4) em função do grau de processamento a ser realizado. Alguns dos produtos relacionados ao balanço energético são o MOD09 (de reflectância de superfície) e o MOD11 (de temperatura da superfície radiométrica). O MOD09 é computado a partir dos níveis $1 \mathrm{~A}$, nas bandas (1 a 7) de estudo da superfície terrestre. O MOD11 ( níveis 2 e 3) tem a recuperação de dados feita a partir do algoritmo Land Surface Temperature (LST), utilizado para recompor pixels que possuam emissividades conhecidas (Anderson et al., 2003).

O sensor AMSR-E representa uma nova fonte de dados para a observação global da umidade do solo. A recuperação de dados sobre a umidade do solo se baseia na inversão dos modelos de transferência radioativa de microondas, que relacionam as variáveis da superfície geofísica à temperatura de brilho observada (Njoku et al., 2003).

\section{Aplicações de modelagem do balan- ço energético superficial}

A Evapotranspiração real pode ser calculada através do algoritmo Surface Energy Balance for Land (SEBAL) desenvolvido por Bastiaanssen (1995). Este modelo tem sido muito utilizado para estimar fluxos de calor e evapotranspiração e se destaca pela sua simplicidade. Além de ser composto por poucas relações empíricas, são utilizados dados multiespectrais com poucas informações referentes à superfície de estudo, como temperatura do ar e velocidade do vento, que são dados facilmente obtidos através das estações meteorológicas (Nicácio, 2008).

No trabalho de Ferraz et al. (2009), o modelo foi utilizado para a estimativa da evapotranspiração real com os seguintes dados de entrada:

1- dados das imagens multiespectrais do 
sensor MODIS (MOD09 e MOD11);

2- dados meteorológicos (temperatura e velocidade dos ventos) adquiridos diretamente de estações meteorológicas.
Mendonça et al. (2009) também utilizaram os mesmos tipos de dados de entrada, obtendo como saída a estimativa do fluxo de calor no solo, conforme o observado na Figura 1:

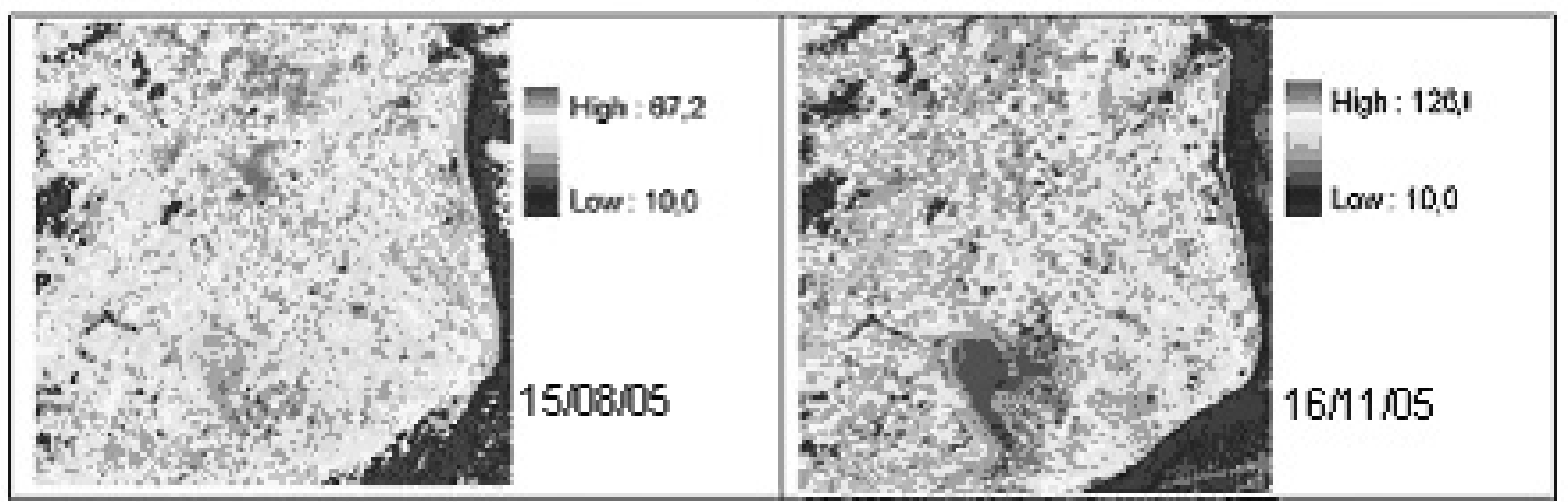

Figura 1. Variação espacial do fluxo de calor no solo, estimado com o algoritmo SEBAL adaptado às imagens do sensor MODIS, na região Norte Fluminense, RJ, nos dias 15/08/05 e 16/11/05, em $\mathrm{Wm}^{-2}$. Fonte: Adaptada de Mendonça et al. (2009).

Renzullo et al. (2008) empregaram técnicas de fusão de dados multisensores e o algoritmo SEBAL para modelar os dados de umidade do solo e temperatura da superfície radiométrica, provenientes dos sensores
ASMR-E e MODIS, respectivamente. Em seguida foram estimados diversos parâmetros biofísicos, entre os quais estão os fluxos de calor latente e sensível no solo, que podem ser, respectivamente, observados na Figura 2.

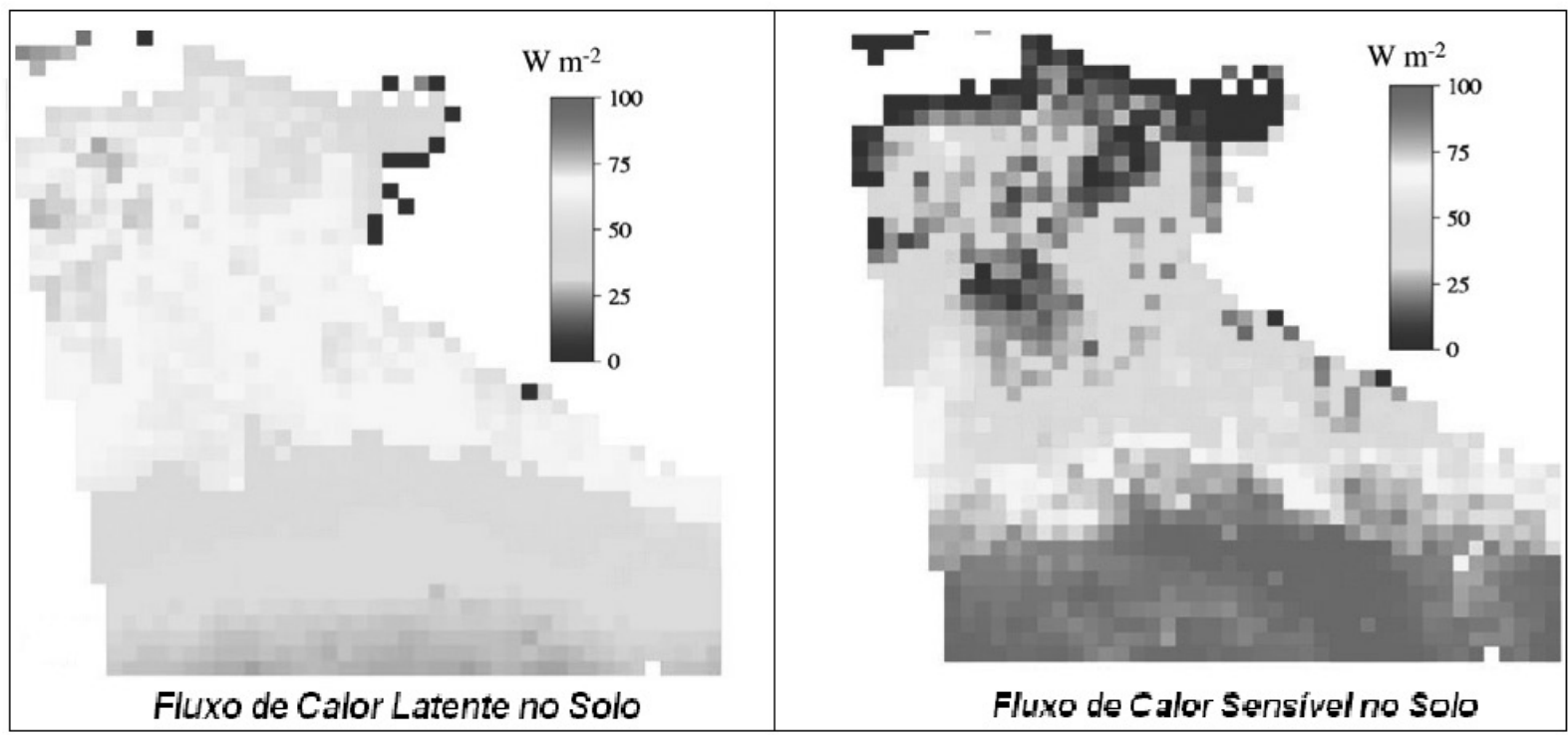

Figura 2. Variação espacial do fluxo de calor latente e sensível no solo, estimado com técnicas de fusão de dados de umidade do solo e temperatura da superfície radiométrica dos sensores ASMR-E e MODIS, na região da savana no norte Australiano, no dia 07/07/04, em $\mathrm{Wm}^{-2}$.

Fonte: Adaptada de Renzullo et al. (2008). 


\section{CONSIDERAÇÕES FINAIS}

Devido às inúmeras dificuldades em se trabalhar com estimativas de parâmetros biofísicos através de medições in loco, e ao advento do sensoriamento remoto, tornou-se indispensável a utilização de técnicas alternativas para a aquisição sistemática e periódica de dados espaciais complementando as informações meteorológicas (Ferraz et al., 2009). O novo satélite HYDROS terá uma configuração mais moderna que o seu antecessor AQUA, apresentando uma melhor resolução espacial e acurácia para contribuir efetivamente com a modelagem de dados. Para isso, o sistema contará com o uso de sensores ativos e passivos em uma única plataforma. $\mathrm{O}$ seu lançamento beneficiará todas as atividades sócio-econômicas sensíveis às mudanças climáticas, entre elas: o gerenciamento dos recursos hídricos, a previsão de safras, o monitoramento de desmatamentos, desastres naturais, inundações, secas e incêndios florestais (Giarolla \& Sano, 2006; Njoku et al., 2004).

Apesar de muitos parâmetros biofísicos encontrarem-se disponíveis através do sensoriamento remoto, é necessário que todos os pressupostos teóricos sejam conhecidos para que as estimativas sejam eficientes. Assim sendo, devido à dificuldade de se obter imagens de sensores orbitais, com uma resolução temporal mais alta, sem a limitação da baixa resolução espacial, é recomendável utilizar sensores com diferentes resoluções (Latorre et al., 2007).

\section{REFERÊNCIAS BIBLIOGRÁFICAS}

ANDERSON, L. O.; LATORRE, M. L.; SHIMABUKURO, Y. E.; ARAI, E.; CARVALHO JR., O. A. de. Sensor MODIS: uma abordagem geral. São José dos Campos: INPE, 2003. 54 p. (INPE-10131-RPQ/752).
BASTIAANSSEN, W. G. M. Regionalization of surface flux densities and moisture indicators in composite terrain. 1995. 273p. PhD Thesis - Wageningen Agricultural University, Wageningen, The Netherlands, 1995.

CHAPIN III, F. S.; MATSON, P. A.; MOONEY, H. A. Terrestrial water and energy balance. In: CHAPIN III, F. S.; MATSON, P. A.; MOONEY, H. A. (Ed.). Principles of Terrestrial Ecosystem Ecology. New York: Springer-Verlag, 2002. cap. 4, p.71-76.

FERRAZ, R. P. D.; MEIRELLES, M. S. P.; JOBAGGY, E.; JONATHAN, M.; COUTINHO, H. L. da C. Utilização de dados MODIS e modelo SEBAL para a estimativa da perda de água por evapotranspiração: Uma proposição metodológica para o monitoramento do impacto da expansão da cultura canavieira na dinâmica hídrica regional. In: SIMPÓSIO BRASILEIRO DE SENSORIAMENTO REMOTO, 14., 2009, Natal. Anais... São José dos Campos: INPE, 2009. p. 175-182. Acesso em: 18 mai. 2009. Online. Disponível em: <marte.dpi.inpe.br/col/dpi. inpe.br/sbsr@80/2008/11.18.12.18/doc/175182.pdf $>$.

FRIEDL, M. A. Forward and inverse modeling of land surface energy balance using surface temperature measurements. Remote Sensing of Environment, v. 79, p. 344-354, Feb 2002.

GIAROLLA, A.; SANO, E. E. Estimativa remota de umidade superficial de solos do cerrado: possibilidades e futuro. In: CONGRESSO BRASILEIRO DE METEOROLOGIA, 14., 2006, Florianopólis. Anais... p. 1-6. Acesso em: 15 mai. 2009. Online. Disponível em: <criatividadecoletiva.net/cbm-files/148d4291198b1fdd7dbebfcdb0d1a36198.pdf>. 
HARTMANN, D. L. The energy balance of the surface. In: HARTMANN, D. L. (Ed.). Global Physical Climatology. San Diego: Academic Press, 1994. cap. 4, p.81-100.

LATORRE, M. L.; CARVALHO JR., O. A. de; SANTOS, J. R. dos; SHIMABUKURO, Y. E. Integração de dados de sensoriamento remoto multiresoluções para a representação da cobertura da Terra utilizando de campos contínuos de vegetação e classificação por árvores de decisão. Revista Brasileira de Geofísica, v. 25, n. 1, p. 63-74, Mar 2007.

MENDONÇA, J. C.; ANDRE, R. G. B.; SOUSA, E. F. de; SILVA, B. B. da. Aplicação do algoritmo SEBAL e imagens MODIS para a estimativa do fluxo do calor do solo (G) na região Norte Fluminense, RJ, Brasil. In: SIMPÓSIO BRASILEIRO DE SENSORIAMENTO REMOTO, 14., 2009, Natal. Anais... São José dos Campos: INPE, 2009. p. 293-300. Acesso em: 18 mai. 2009. Online. Disponível em: <marte.dpi.inpe.br/col/ dpi.inpe.br/sbsr@80/2008/ 11.17.21.24.45/ doc/293-300.pdf>.

NICÁCIO, R. M. Evapotranspiração real e umidade do solo usando dados de sensores orbitais e a metodologia SEBAL na bacia do rio São Francisco. 2008. 320 p. Tese (Doutorado em Engenharia Civil) - Uni- versidade Federal do Rio de Janeiro, Rio de Janeiro, 2008.

NJOKU,E. G.; SPENCER, M.; MCDONALD, K.; SMITH, J. The Hydros Mission: Requirements and design. In: IEEE AEROSPACE CONFERENCE, 2004. Proceedings... v. 2, p. 1000-1007. (AERO.2004.1367701). Acesso em 26 mai. 2009. Online. Disponível em: <ieeexplore.ieee.org/stamp/ stamp. jsp?arnumber $=01367701>$.

NJOKU, E. G.; JACKSON, T. J.; LAKSHMI, V.; CHAN, T. K.; NGHIEM, S. V. Soil Moisture Retrieval From AMSR-E. IEEE Transactions on Geoscience and Remote Sensing, v. 41, n. 2, p. 215-229, Feb 2003.

RENZULLO, L. J.; BARRETT, D. J.; MARKS, A. S.; HILL, M. J.; GUERSCHMAN, J. P.; MU, Q.; RUNNING, S. W. Multi-sensor model-data fusion for estimation of hydrologic and energy flux parameters. Remote Sensing of Environment, v. 112, p. 1306-1319, Apr 2008.

ROSENBERG, N. J.; BLAD, B. L.; VERNA, S. B. The radiation balance. In: ROSENBERG, N. J.; BLAD, B. L.; VERNA, S. B. (Ed.) Microclimate: The biological environment. 2 ed. New Jersey: Wiley-Interscience, 1983. cap. 1, p. 5-58. 